\title{
Accurate rest frequencies for propargylamine in the ground and low-lying vibrational states ${ }^{\star}$
}

\author{
C. Degli Esposti ${ }^{1}$, L. Dore ${ }^{1}$, C. Puzzarini ${ }^{1}$, M. Biczysko², J. Bloino ${ }^{3,4}$, L. Bizzocchi ${ }^{5}$, \\ V. Lattanzi ${ }^{5}$, and J.-U. Grabow ${ }^{6}$
}

\author{
${ }^{1}$ Dipartimento di Chimica "Giacomo Ciamician", Università di Bologna, via Francesco Selmi 2, 40126 Bologna, Italy \\ e-mail: luca.dore@unibo.it; claudio.degliesposti@unibo.it \\ ${ }^{2}$ International Centre for Quantum and Molecular Structures, College of Sciences, Shanghai University, 99 Shangda Road, \\ Shanghai 200444, China \\ ${ }^{3}$ Consiglio Nazionale delle Ricerche, Istituto di Chimica dei Composti OrganoMetallici (ICCOM-CNR), UOS di Pisa, Area della \\ Ricerca CNR, Via Giuseppe Moruzzi 1, 56124 Pisa, Italy \\ ${ }^{4}$ Scuola Normale Superiore, Piazza dei Cavalieri 7, 56126 Pisa, Italy \\ 5 Centre for Astrochemical Studies, Max-Planck-Institut für extraterrestrische Physik, Gießenbachstraße 1, \\ 85749 Garching bei München, Germany \\ ${ }^{6}$ Institut für Physikalische Chemie und Elektrochemie, Gottfried-Wilhelm-Leibniz-Universität, Lehrgebiet A, \\ Hannover 30167 Germany
}

Received 31 January 2018 / Accepted 16 April 2018

\begin{abstract}
Context. To date, several complex organic molecules have been detected in the interstellar medium, and they have been suggested as precursors of biologically important species. Propargylamine $\left(\mathrm{HC} \equiv \mathrm{C}-\mathrm{CH}_{2}-\mathrm{NH}_{2}\right)$ is structurally similar to a number of other organic molecules which have already been identified by radioastronomy, making it a good candidate for astrophysical detection.

Aims. This work provides accurate rest frequencies of propargylamine, from the centimeter-wave to the submillimeter-wave region, useful to facilitate the detection of this molecule in the interstellar medium.

Methods. An extensive laboratory study of the rotational spectrum of propargylamine has been performed using a pulsed-jet Fourier Transform Microwave (FTMW) spectrometer (7-19 GHz frequency range) and a frequency modulation microwave spectrometer $(75-560 \mathrm{GHz})$. Several hundred rotational transitions of propargylamine were recorded in the ground and three lowest excited vibrational states. The experiments were supported by high-level ab initio computations, mainly employed to characterize the vibrational state structure and to predict spectroscopic parameters unknown prior to this study.

Results. The measured transition frequencies yielded accurate rotational constants and the complete sets of quartic and sextic centrifugal distortion constants for propargylamine in its vibrational ground state. ${ }^{14} \mathrm{~N}$-nuclear quadrupole coupling constants were also determined. Rotational and quartic centrifugal distortion constants were also obtained for the low-lying excited states $v_{13}=1\left(A^{\prime}\right)$, $v_{20}=1\left(A^{\prime \prime}\right)$, and $v_{21}=1\left(A^{\prime \prime}\right)$. The $a$-type Coriolis resonance which couples the $v_{13}=1$ and $v_{21}=1$ levels was analyzed.

Conclusions. The determined spectroscopic constants allowed for the compilation of a dataset of highly accurate rest frequencies for astrophysical purposes in the millimeter and submillimeter regions with $1 \sigma$ uncertainties that are smaller than $0.050 \mathrm{MHz}$, corresponding to $0.03 \mathrm{~km} \mathrm{~s}^{-1}$ at $500 \mathrm{GHz}$ in radial equivalent velocity.
\end{abstract}

Key words. molecular data - methods: laboratory: molecular - techniques: spectroscopic - radio lines: ISM

\section{Introduction}

Over the last few years, the formation of astronomical complex organic molecules (COMs) in the interstellar medium (ISM) has received considerable attention due to their potential prebiotic role. Understanding how the building blocks of biological molecules were formed in abiotic environments (being either the primitive Earth or the ISM) is in fact an extremely topical issue. In particular, amines $\left(\mathrm{RNH}_{2}\right)$ might play a key role as intermediates in the formation of astrobiologically important molecules, such as amino acids (Holtom et al. 2005; Bossa et al. 2008; Lee et al. 2009; Kim \& Kaiser 2011). Almost 200 molecules have been detected in space, thus implying that even in the harsh conditions of the ISM, a rich chemistry takes place. Focusing

\footnotetext{
* The full Table 4 is only available at the CDS via anonymous ftp to cdsarc.u-strasbg.fr $(130.79 .128 .5)$ or via http://cdsarc.u-strasbg.fr/viz-bin/qcat?]/A+A/615/A176
}

on planetary atmospheres, Titan has the most active one within the Solar System, from a chemical perspective. It has been shown that, starting from the simple parent molecules $\mathrm{N}_{2}$ and $\mathrm{CH}_{4}$, small $\mathrm{N}$-containing species up to large macromolecules (with masses up to 3000 Dalton) are produced in the upper and most rarefied region of the atmosphere (Balucani 2012; Ali et al. 2015, and relevant references therein). Extraterrestrial gaseous species are mainly identified via observations of their rotational signatures, knowledge of which is derived from laboratory experiments that in turn can be effectively supported and extended by theoretical predictions (Barone et al. 2015b; Biczysko et al. 2017).

Propargylamine $\left(\mathrm{HC} \equiv \mathrm{C}-\mathrm{CH}_{2}-\mathrm{NH}_{2}\right)$ is a $\mathrm{N}$-containing derivative of acetylene, which is structurally related to other astrophysically relevant species such as aminoacetonitrile $\left(\mathrm{N} \equiv \mathrm{C}-\mathrm{CH}_{2}-\mathrm{NH}_{2}\right.$; Belloche et al. 2008) and methylacetylene $\left(\mathrm{CH}_{3} \mathrm{C} \equiv \mathrm{CH}\right.$; Snyder \& Bull 1973) which have been detected in 


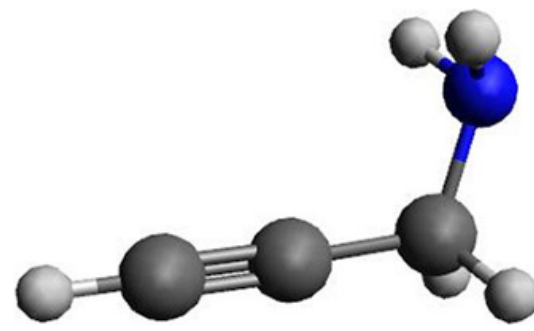

Fig. 1. Structure of trans-propargylamine. Blue atom is nitrogen, darkgray are carbon, and light-gray are hydrogen.

molecular clouds. The formation routes of propargylamine in the ISM have still to be investigated in detail; nonetheless, there are a number of reasons to regard this COM as a spectroscopic target of potential astrochemical interest.

It is nowadays accepted that reactions on dust-grain surface play a major role in building the chemical complexity in the ISM. Hydrogenation, in particular, is a very efficient process on grains due to the high mobility of $\mathrm{H}$ atoms on these surfaces at $T<20 \mathrm{~K}$ (Tielens \& Hagen 1982; Tielens \& Charnley 1997; Fuchs et al. 2009). This reaction tends to saturate multiple bonds of the simple molecules that are frozen onto dust-grain mantles during the cold pre-collapse phases of star formation. The resulting products are then released into gas phase at the proto-star stage, when the mantles are evaporated by the heating produced by the central source (Ceccarelli et al. 2001). Surface hydrogenation of iced $\mathrm{CO}$ is postulated to be the formation route of $\mathrm{CH}_{3} \mathrm{OH}$ and other O-bearing COMs (e.g., Watanabe \& Kouchi 2002; Bisschop et al. 2007; Garrod et al. 2007; Ioppolo et al. 2010). Similarly, a related series of $\mathrm{H}$-addition reactions could take place on grain surfaces to generate the simplest member of the amine family, $\mathrm{CH}_{3} \mathrm{NH}_{2}$, starting from the very abundant HCN species (e.g., Theulé et al. 2011), and a natural extension of this reasoning lead to infer that propargyl-imine and propargylamine could be obtained through $2 \mathrm{H}-$ and $4 \mathrm{H}$ - partial saturation of $\mathrm{HC}_{3} \mathrm{~N}$, a carbon chain which proved to be ubiquitous in the ISM (Bizzocchi et al. 2017).

Two conformers (trans and gauche forms) can exist for propargylamine, but only the rotational spectrum of the most stable trans form (whose structure is shown in Fig. 1) has been detected by microwave spectroscopy, just as in the case of the isoelectronic aminoacetonitrile molecule.

Rotational lines of trans-propargylamine $\left(C_{s}\right.$ symmetry group) were first observed by Bolton et al. (1968). A subsequent study in the 8-40 GHz frequency range (Cervellati et al. 1977) allowed the determination of the rotational constants of nine isotopically substituted species, from which a reliable molecular structure could be calculated. Additionally, the dipole moment of trans-propargylamine was determined by Stark effect measurements (the values $\mu_{a}=0.586 \mathrm{D}$ and $\mu_{b}=0.445 \mathrm{D}$ being derived), but the very limited frequency range over which the rotational spectrum was studied prevented from obtaining very accurate values of the centrifugal distortion constants. Although Cervellati et al. (1977) were able to observe rotational lines of the ${ }^{13} \mathrm{C}$ and ${ }^{15} \mathrm{~N}$ containing species in natural abundance, the search for transitions of the gauche conformer was unsuccessful. This non-detection, combined with a theoretical evaluation of the dipole moment components of the gauche form, made it possible to estimate a lower limit of approximately $2600 \mathrm{cal} \mathrm{mol}^{-1}$ (corresponding to $910 \mathrm{~cm}^{-1}$ ) for the energy difference between the gauche and the trans forms.

The low-resolution, gas-phase infrared spectrum of propargylamine (Hamada et al. 1984) led to the assignment of all the
21 fundamental bands of the trans-conformer $\left(A^{\prime}\right.$ symmetry from $v_{1}$ to $v_{13}, A^{\prime \prime}$ symmetry from $v_{14}$ to $\left.v_{21}\right)$. The recorded spectrum did not reveal any prominent absorption band assignable to the gauche form, for which a room temperature abundance not larger than $3 \%$ was guessed, corresponding to an energy difference of at least $810 \mathrm{~cm}^{-1}$. Later, the $v_{8}$ and $v_{9}$ fundamental bands of propargylamine were studied in the $900-1100 \mathrm{~cm}^{-1}$ region using a $2 \mathrm{MHz}$-resolution electric resonance optothermal molecular beam spectrometer with a microwave sideband $\mathrm{CO}_{2}$ laser (Andrews et al. 1994). In both bands, tunneling doublets with 3:1 relative intensities were observed, indicative of an amine proton interchange (Andrews et al. 1998).

In this work, the pure rotational spectrum of propargylamine in its vibrational ground state has been thoroughly reinvestigated. We have measured 256 new rotational lines in the millimeter-wave (mm-wave) and submillimeter-wave (submmwave) regions up to $554 \mathrm{GHz}$ and a rotational quantum number $J$ of 65 , allowing for a very comprehensive centrifugal distortion analysis. In addition to high-frequency measurements, the centimeter-wave (cm-wave) spectrum of propargylamine in the vibrational ground state has been investigated using a Fabry-Perot-type resonator pulsed-jet Fourier Transform microwave (FTMW) spectrometer. Thirty-four hyperfine structure components belonging to low- $J$ rotational transitions have been recorded in the $7-19 \mathrm{GHz}$ range, and their analysis yielded accurate values of the ${ }^{14} \mathrm{~N}$ electric quadrupole coupling constants.

With the increased sensitivity and angular resolution of new observatories, with particular mention to the Atacama Large Millimeter/submillimeter Array (ALMA), the number of unidentified lines in the astronomical spectra has grown, especially in those of hot cores associated with star formation. Among the unidentified lines, a good fraction is thought to be due to rotational transitions in the low-lying excited vibrational states of a relatively small number of molecules. Also for this reason, the rotational spectra of propargylamine in excited vibrational states have been recorded and analyzed for the first time. Over 350 lines belonging to the three lowest excited states have been assigned in the 75-360 GHz frequency range, spanning $J$ values from 7 to 42 . From line intensity ratio measurements it was possible to approximately evaluate the vibrational energies of the observed states, which were found in partial disagreement with previous assignments (Hamada et al. 1984).

The spectroscopic characterization of propargylamine has been supported by a well-tested hybrid coupled-cluster/density functional theory (CC/DFT) protocol (Barone et al. 2013, 2014b, 2015a; Puzzarini et al. 2014).

\section{Experimental and computational details}

\subsection{Experiment}

The rotational spectrum of propargylamine was investigated using a source-modulation $\mathrm{mm}$-/submm-wave spectrometer located in Bologna (Dipartimento di Chimica "Giacomo Ciamician") and a Fabry-Perot-type resonator pulsedjet FTMW spectrometer at the Gottfried-Wilhelm-Leibniz Universität in Hannover (Institut für Physikalische Chemie and Elektrochemie). Commercial samples of propargylamine (Sigma Aldrich) were used for the measurements without further purification. The mm- and submm-wave lines were recorded in selected frequency regions between 75 and $555 \mathrm{GHz}$ using the spectrometer already employed to record the spectra of other stable (Degli Esposti et al. 2017) and short-lived molecules 
(Degli Esposti et al. 2014; Bizzocchi et al. 2016) of astrophysical interest. Gunn-diode oscillators were used as main radiation sources to cover the fundamental frequency range $75-125 \mathrm{GHz}$, and higher frequencies were generated using passive harmonic multiplication. The oscillators were phase-locked through a suitable locking chain to a computer-controlled frequency synthesizer, which was referenced to an external rubidium frequency standard. The source was frequency modulated at $6 \mathrm{kHz}$ and phase sensitive detection at $2 f$ was employed, so that the second derivative of the actual spectrum profile was recorded. Schottky-barrier diodes and a liquid-helium-cooled $\mathrm{InSb}$ bolometer were used as detectors in the mm- and submmwave regions, respectively. A 3.5-m-long free-space absorption cell filled with propargylamine vapor at pressures of $1.3-2.7 \mathrm{~Pa}$ was employed for the measurements. The spectrometer used for FTMW supersonic expansion measurements has been described in detail previously (Grabow \& Stahl 1990; Grabow et al. 1996). The sample was prepared by injecting a few drops of propargylamine into a 5 liter steel cylinder that was then filled with Argon at a total pressure of $500 \mathrm{kPa}$. Given that propargylamine has a vapor pressure of $10.3 \mathrm{kPa}$ at $300 \mathrm{~K}$, the resulting gaseous mixture has a volume fraction of approximately $2 \%$. The measurements were performed in the $7-19 \mathrm{GHz}$ frequency interval, employing a stagnation pressure of $50-150 \mathrm{kPa}$ with a nozzle repetition rate of $20 \mathrm{~Hz}$.

\subsection{Computations}

The computational strategy relies on coupled-cluster (CC) calculations (at the $\mathrm{CC}$ singles and doubles augmented by a perturbative treatment of triple excitations level, $\operatorname{CCSD}(\mathrm{T})$; Raghavachari et al. 1989) of equilibrium geometries, electric properties, and quadratic force constants in a normal-mode representation (i.e., harmonic frequencies), also considering extrapolation to the complete basis set (CBS) limit and core-valence correlation $(\mathrm{CV})$ contributions. These results are then complemented by density functional theory (DFT) evaluations of the anharmonic contributions (Barone et al. 2014a; Bloino et al. 2015, 2016) employing the double hybrid B2PLYP functional (Grimme 2006; Biczysko et al. 2010) including the D3BJ dispersion corrections (Grimme et al. 2010, 2011) in conjunction with the aug-cc-pVTZ basis set.

For the equilibrium structure determination, from which the equilibrium rotational constants are straightforwardly derived, a composite approach was used based on the additivity of relevant contributions at a gradient level (Heckert et al. 2005, 2006), as implemented in the CFOUR package ${ }^{1}$ Stanton et al. (2011). The three contributions taken into account are: the HartreeFock self-consistent-field (HF-SCF) energy extrapolated to the complete basis-set (CBS) limit, the valence correlation energy at the $\operatorname{CCSD}(\mathrm{T})$ (Raghavachari et al. 1989), extrapolated to the CBS limit as well, and the core-valence $(\mathrm{CV})$ correlation correction. The correlation-consistent cc-p $(\mathrm{C}) \mathrm{VnZ}(n=\mathrm{T}, \mathrm{Q}, 5)$ basis sets (Dunning Jr. 1989; Woon \& Dunning Jr. 1995) were employed. The overall best-estimated geometry, accounting for the extrapolation to the CBS limit and CV correction, is shortly denoted in the following as $\operatorname{CCSD}(\mathrm{T}) / \mathrm{CBS}+\mathrm{CV}$. A similar composite scheme was also employed for obtaining the best-estimated equilibrium values of the electric dipole moment components. The only difference lies in the inclusion of the contribution of diffuse functions in the basis set.

\footnotetext{
1 http://www.cfour.de
}

The best-estimated harmonic force field was evaluated by means of the following composite scheme (Barone et al. 2013):

$\omega_{\text {best }}=\omega(\mathrm{CC} / \mathrm{TZ})+\Delta \omega(\mathrm{MP} 2 / \mathrm{CBS})+\Delta \omega(\mathrm{CV})+\Delta \omega(\mathrm{aug})$,

where the harmonic frequencies at the $\operatorname{CCSD}(\mathrm{T}) / \mathrm{cc}-\mathrm{pVTZ}$ $(\omega(\mathrm{CC} / \mathrm{TZ}))$ level are corrected to account for the extrapolation to the CBS limit $(\Delta \omega(\mathrm{MP} 2 / \mathrm{CBS}))$, core-valence correction $(\triangle \omega(\mathrm{CV}))$, and contribution of diffuse functions in the basis set $(\Delta \omega(\mathrm{aug}))$ using second-order Møller-Plesset perturbation theory (MP2; Møller \& Plesset 1934). Harmonic force constants were obtained at the corresponding optimized geometry using analytic second derivatives, as implemented in CFOUR (Stanton et al. 2011; Gauss \& Stanton 1997). As a byproduct, bestestimated quartic centrifugal-distortion and nitrogen-quadrupole coupling constants were obtained from the best-estimated harmonic force field and the corresponding geometry optimizations, respectively.

Anharmonic contributions to vibrational energies and spectroscopic constants were calculated at the second order of the vibrational perturbation theory (VPT2; Nielsen 1951; Krohn et al. 1974. This includes vibrational corrections to the rotational and nitrogen-quadrupole coupling constants and to the electric dipole moment, as well as calculation of sextic centrifugal distortion constants. For the vibrational states of interest in the present study, no resonances related to the VPT2 formalism were found, and therefore the standard equations were used.

The cubic and semi-diagonal quartic force field needed for the VPT2 calculations was obtained from numerical differentiation of displaced analytic harmonic force constants along the normal coordinates computed at the B2PLYP-D3BJ/augcc-pVTZ level. The hybrid force field was obtained in a normal-coordinate representation by adding the cubic and quartic B2PLYP-D3BJ/aug-cc-pVTZ force constants to the bestestimated harmonic frequencies. When the normal modes computed at different levels of theory are very similar, as in the present case, DFT cubic and quartic force constants can be used without any transformation. The DFT and VPT2 computations were done with the Gaussian 16 suite of quantum chemical programs (Frisch et al. 2016).

Although the aim of this study is to provide accurate rest frequencies for the most stable conformer of propargylamine, that is, the trans form, a computational investigation of the molecular structure and dipole moment for the gauche conformer was also carried out at the B2PLYP-D3BJ/aug-cc-pVTZ level, mainly to check the reliability of the conclusions drawn by Cervellati et al. (1977) about the energy of the undetected gauche form. The following values of the dipole moment components of the two isomers were obtained at the same level of theory: $\mu_{a}=0.5215 \mathrm{D}$ and $\mu_{b}=0.4351 \mathrm{D}$ for the trans form, and $\mu_{a}=0.7334 \mathrm{D}$, $\mu_{b}=0.7265 \mathrm{D}$, and $\mu_{c}=1.0480 \mathrm{D}$ for the gauche form. Using the newly evaluated theoretical values of the $\mu_{a}$ components, a lower limit of approximately $3500 \mathrm{cal} \mathrm{mol}^{-1}\left(1220 \mathrm{~cm}^{-1}\right)$ can be estimated for the energy difference between the two conformers.

\section{Observed spectra and analysis}

\subsection{Ground state}

Trans-propargylamine is a near-prolate asymmetric-top molecule $(\kappa=-0.966)$ of $C_{S}$ symmetry, with non-zero dipole moment components along the $a$ and $b$ principal axes that define the symmetry plane of the molecule (Cervellati et al. 1977). Regarding the vibrational ground state, initial 
assignments in the mm-wave region were guided by the spectroscopic constants available from the previous $\mathrm{cm}$-wave investigation (Cervellati et al. 1977). ${ }^{a} R$-branch transitions and additional $b$-type lines were first recorded in the $100 \mathrm{GHz}$ region. New predictions, based on improved rotational and centrifugal distortion constants, were then used to guide measurements in the submm-wave region, at frequencies up to $554 \mathrm{GHz}$. In this way our measurements probed rotational energy levels in the $J$ interval from 5 to 65 and the $K_{a}$ interval from 0 to 26 . Small splittings due the ${ }^{14} \mathrm{~N}$ nuclear spin were observed for a few ${ }^{a} R$-type transitions with high $K_{a}$ values measured around $100 \mathrm{GHz}$.

To improve the precision of the hyperfine-structure parameters, complementary measurements were performed also in the $7-19 \mathrm{GHz}$ range on low- $J$ lines, which were observed in supersonic expansion and with fully resolved ${ }^{14} \mathrm{~N}$-nuclear quadrupole hyperfine structure. Seven rotational transitions were recorded at sub-Doppler resolution by FTMW spectroscopy: they include the $a$-type $J=1 \leftarrow 0$ and $J=2 \leftarrow 1$ transitions, plus three low-lying $b$-type transitions with $K_{a}=0,1$, so that a total number of 34 hyperfine-component frequencies could be measured with high precision.

The complete dataset for the ground state was therefore assembled by combining the $\mathrm{mm}$ - and submm-wave transition frequencies from the present work, with previous hyperfineunresolved cm-wave lines (Cervellati et al. 1977), and with newly measured hyperfine resolved frequencies for low- $J$ transitions. Different weights $w_{i}=1 / \sigma_{i}^{2}$ were used for the various datasets in order to take into account differences in measurement precision: $\sigma_{i}$ values in the range from 10 to $100 \mathrm{kHz}$ were assigned to the newly measured $\mathrm{mm}$ - and submm-wave transitions, depending on the signal-to-noise ratio $(\mathrm{S} / \mathrm{N})$ and the width of each line, accidental overlaps of lines, as well as the frequency uncertainty produced by unresolved ${ }^{14} \mathrm{~N}$ quadrupole components. For the present FTMW measurements, we adopted a conservative value of $\sigma_{i}=5 \mathrm{kHz}$ to take into account the asymmetric line broadening produced by the blending of hyperfine components due to the nuclear spins of the five hydrogen atoms. The previous Stark-modulation measurements (Cervellati et al. 1977) were given with an average uncertainty of $\sigma_{i}=85 \mathrm{kHz}$.

The resulting dataset was analyzed using Pickett's SPFIT program (Pickett 1991), adopting Watson's $S$-reduced Hamiltonian in its $I^{r}$ representation (Watson 1977). The standard coupling scheme $F=J+I$, between the rotational angular momentum $J$ and the ${ }^{14} \mathrm{~N}$-nuclear spin $I$, was used to analyze the observed hyperfine-structure components.

A large set of centrifugal distortion parameters was fitted for the vibrational ground state; in detail, all quartic and sextic centrifugal-distortion constants could be derived, as well as a few octic distortion terms. Likewise, the diagonal elements of the nuclear electric quadrupole tensor in the principal axis system could be precisely determined. The results are reported in Table 1 together with the corresponding theoretical results. We note that the computed rotational and quartic centrifugal-distortion constants agree well with their experimental counterparts, with deviations below $0.3 \%$ and $\simeq 5 \%$, respectively. For sextic centrifugal terms, larger discrepancies, that is, on the order of $15 \%-20 \%$, were expected, but still the accuracy was sufficient for a satisfactory prediction of the experiment. These findings are consistent with the literature on this topic (Puzzarini et al. 2008; Puzzarini 2013). A good agreement can also be noted for nitrogen-quadrupole coupling constants and dipole moment components. In Table 1, the ground-state rotational and centrifugal distortion constants

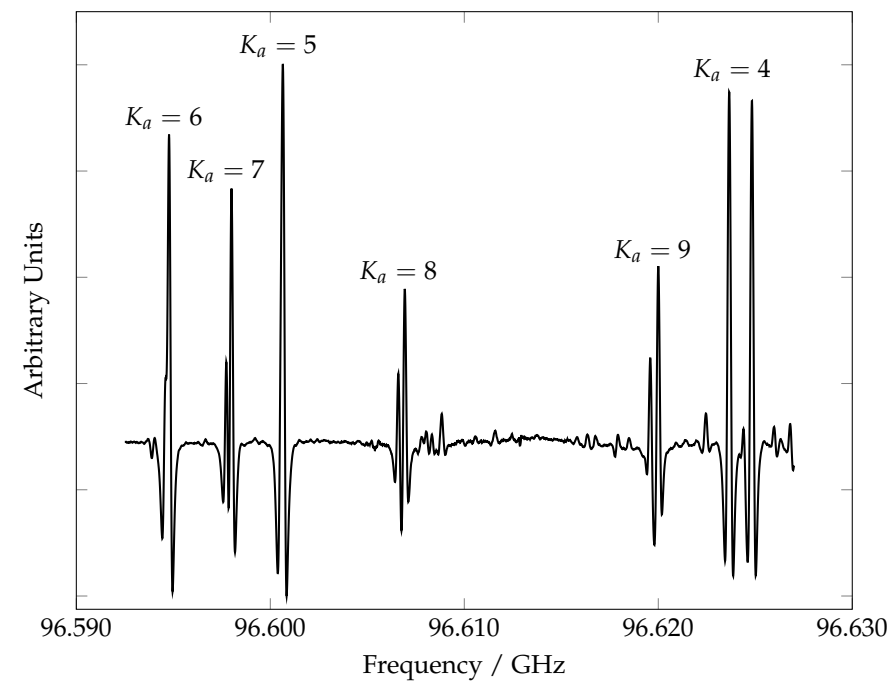

Fig. 2. Portion of the $J=11 \leftarrow 10$, $a$-type band of propargylamine in the $v_{13}=1$ state. Splittings caused by electric quadrupole coupling are observable for $K_{a}=7,8$, and 9 . The asymmetry doublet of the $K_{a}=4$ transition is also visible.

determined for aminoacetonitrile (Motoki et al. 2013) are also reported for comparison. As already stated in the Introduction, propargylamine is structurally very similar to aminoacetonitrile, and similar rotational parameters are therefore expected. This is confirmed by an inspection of Table 1 .

\subsection{Excited states}

Rotational transitions belonging to three low-lying excited vibrational states were also observed and analyzed. The three lowest vibrational modes were assigned by IR spectroscopy (Hamada et al. 1984) in the following way: $v_{13}$ at $230 \mathrm{~cm}^{-1}\left(A^{\prime}\right.$ symmetry, in-plane $\mathrm{C} \equiv \mathrm{C}-\mathrm{C}$ linear bending), $v_{21}$ at $334 \mathrm{~cm}^{-1}$ ( $A^{\prime \prime}$ symmetry, $-\mathrm{NH}_{2}$ torsion), and $v_{20}$ at $339 \mathrm{~cm}^{-1}$ ( $A^{\prime \prime}$ symmetry, out-of-plane $\mathrm{C} \equiv \mathrm{C}-\mathrm{C}$ linear bending). At room temperature, all these states are sufficiently populated to ensure the detection of the respective rotational spectra. We started the search for the excited-state rotational lines in the $75-110 \mathrm{GHz}$ frequency range. Near each ${ }^{a} R$ branch of the ground state ( $J$ from 8 to 11 ) we were able to identify three different sequences of lines with the same $K_{a}$ structure, but with reduced intensities, as expected for vibrational satellites produced by low-lying vibrational states. An example is provided in Fig. 2, showing the compact $K_{a}$ sequence of the central part of the $J=11 \leftarrow 10 a$-type band of the $v_{13}=1$ state. These characteristic sequences of lines, always observed at frequencies higher than those of the ground state, were the fingerprints used to identify the various excited states.

Intensity ratio measurements provided approximate values of the excited-state vibrational energies, which were 185, 233, and $327 \mathrm{~cm}^{-1}$, respectively, not fully consistent with the old IR data. The measurements for the three excited states were then extended up to $360 \mathrm{GHz}$, including both $a$ - and $b$-type transitions. The rotational lines belonging to the state of highest energy $\left(v_{20}=1\right)$ could be very well fitted using the standard semi-rigid Hamiltonian, with all sextic centrifugal-distortion constants fixed to the ground-state values. The results are listed in Table 2, together with the rotational constants predicted by quantum-chemical calculations. Conversely, this procedure gave unsatisfactory results for the two states of lower energy. Indeed, large deviations, opposite in sign, were observed for ${ }^{a} R$ lines with 
Table 1. Ground-state spectroscopic constants of trans-propargylamine (present work) compared with those of trans-aminoacetonitrile (Motoki et al. 2013).

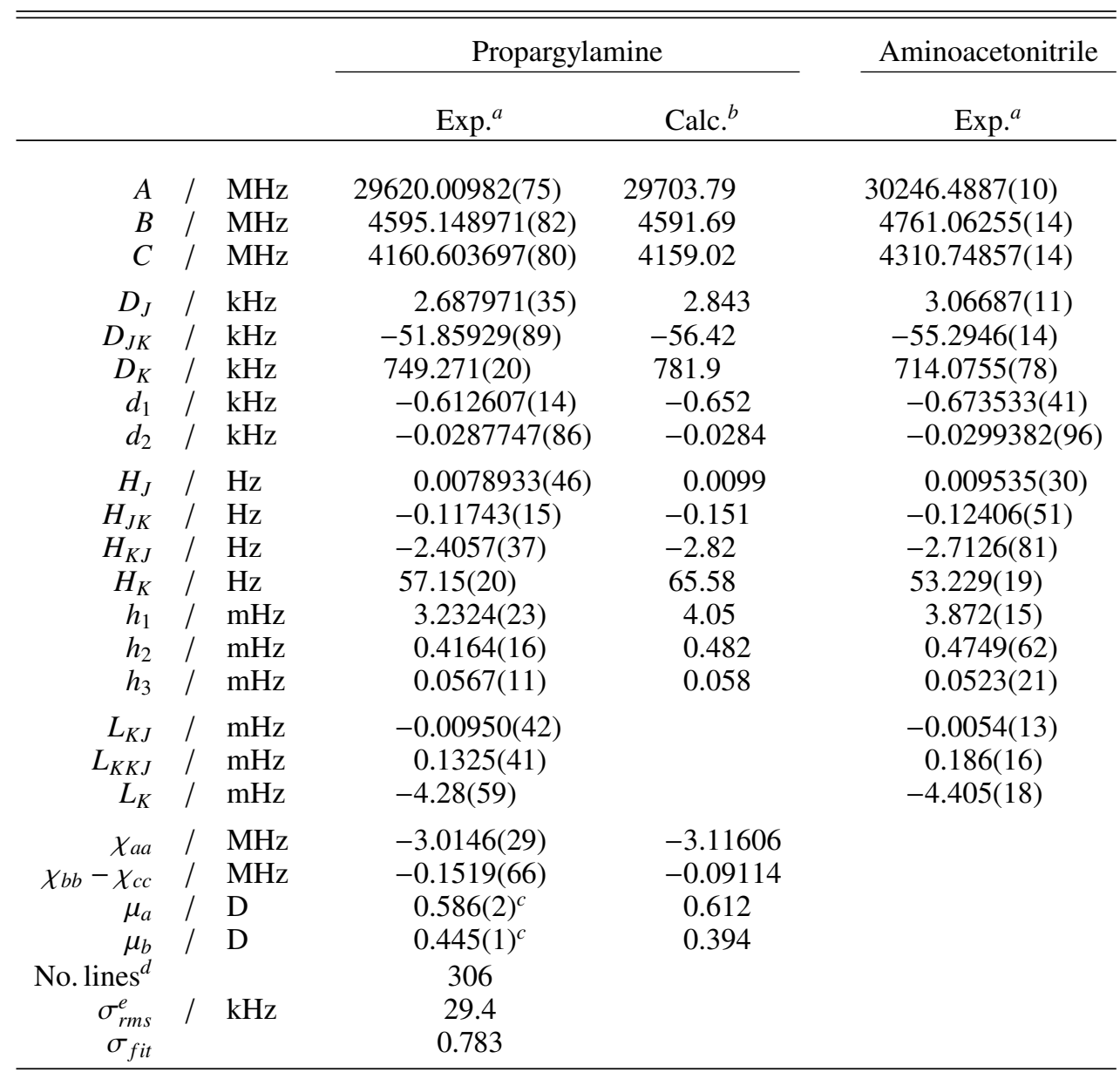

Notes. ${ }^{(a)}$ The quantities in parentheses are standard errors $(1 \sigma)$ in units of the last quoted digit. ${ }^{(b)}$ Rotational, quadrupole-coupling constants, and dipole moment components: best-estimated equilibrium parameters augmented by vibrational corrections at the B2PLYP-D3BJ/aug-cc-pVTZ level. Quartic and sextic centrifugal-distortion constants from the hybrid CC/DFT force field. See text. ${ }^{\left({ }^{(}\right)}$From Cervellati et al. (1977). ${ }^{(d)}$ Number of distinct line frequencies analyzed. ${ }^{(e)}$ RMS error of residuals.

$K_{a}>J / 2$. Moreover, the fittings produced strongly diverging values of the quartic centrifugal-distortion constants $D_{K}$ and $d_{2}$. This was a clear indication of the existence of some rovibrational interaction between the two states of lower energy, as it was observed for aminoacetonitrile (Degli Esposti et al. 2017).

Since trans-propargylamine is a non-planar molecule of $C_{s}$ symmetry (the $c$ axis being perpendicular to the symmetry plane) its vibrations must belong to the $A^{\prime}$ or $A^{\prime \prime}$ irreducible representations. Two fundamental levels of the same symmetry are connected by the $c$-axis term included in the $H_{21}$ Coriolis operator, while two states of different symmetry can be coupled by $a$ and $b$-axis Coriolis operators (Papoušek \& Aliev 1982).

As a matter of fact, the quality of the fits could be greatly improved by assuming the existence of an $a$-type Coriolis interaction, which demonstrates that the two lowest vibrational states of trans-propargylamine must be of different symmetry. The two states were considered coupled through the off-diagonal $G_{a} P_{z}$ Coriolis operator, and the transition frequencies of the two states were simultaneously analyzed by also fitting the Coriolis coupling coefficient $G_{a}$ and the vibrational energy difference between the two states. The standard deviation of the fit then decreased from $2 \mathrm{MHz}$ to $50 \mathrm{kHz}$, even if small systematic residuals still remained, indicating the need for improvement of the coupling model. No significant effect was obtained by considering either a $b$-axis Coriolis interaction or higher-order terms deriving from $H_{22}$ (Papoušek \& Aliev 1982), while a further improvement of the fit was achieved by taking into account small dependencies of $G_{a}$ on the rotational quantum numbers $J$ and $K$.

The best results were obtained adopting an off-diagonal Coriolis operator of the form $\left(G_{a}+G_{a}^{J K} P^{2} P_{z}^{2}\right) P_{z}$ and fitting the $G_{a}^{J K}$ parameter: this led to a standard deviation of the fit smaller than $20 \mathrm{kHz}$. It must be noted that the same improvement could be alternatively obtained by fitting the sextic distortion constants $H_{K J}$ of both resonant states, but in this case two very unreliable values, differing by more than a factor of six, are derived. On the contrary, no significant improvement was obtained by releasing the $G_{a}^{J}$ and $G_{a}^{K}$ coefficients, which were poorly determined and were therefore held fixed. $G_{a}^{J}$ was constrained to the fairly accurate value which was determined for aminoacetonitrile (Degli Esposti et al. 2017), while $G_{a}^{K}$ was simply fixed to zero. The results of the least-squares fits for these two interacting states are presented in Table 2, where the rotational constants obtained by quantum-chemical calculations are also reported. No important anomaly appears in the values of the centrifugal distortion constants determined for the two 
Table 2. Spectroscopic constants determined for the $v_{13}=1$ and $v_{21}=1$ resonant states and for the $v_{20}=1$ unperturbed state of transpropargylamine.

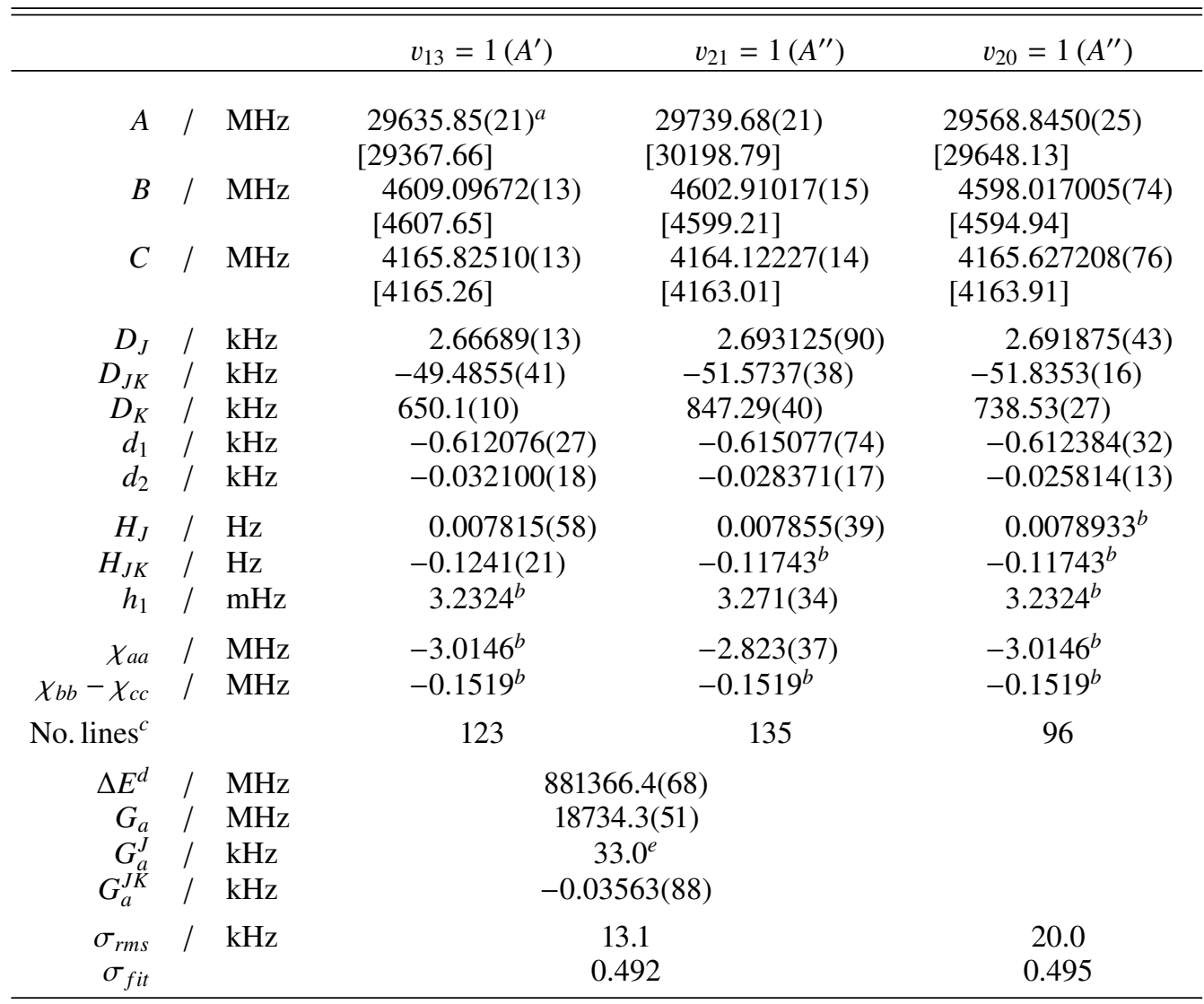

Notes. ${ }^{(a)}$ The quantities in parentheses are standard errors $(1 \sigma)$ in units of the last quoted digit. Theoretical values are given between squared brackets. ${ }^{(b)}$ Parameter fixed at the ground-state value, likewise for the sextic centrifugal distortion constants not explicitly reported in this table. ${ }^{(c)}$ Number of distinct line frequencies analyzed for each vibrational state. ${ }^{(d)}$ Vibrational energy difference between the $v_{21}=1$ upper state and the $v_{13}=1$ lower state. ${ }^{(e)}$ Parameter fixed at the value obtained for aminoacetonitrile (Degli Esposti et al. 2017).

resonant states, but we note that the fitted $D_{K}$ constants still differ considerably, in a symmetrical way, from the ground-state value (ca. $\pm 13 \%$ ). This could indicate an incomplete correction of resonance effects (totally uncorrected values were $D_{K}=434 \mathrm{kHz}$ for $v_{13}$ and $D_{K}=1307 \mathrm{kHz}$ for $\left.v_{21}\right)$. $D_{K}$ values much closer to that of the ground state can be obtained by fixing the centrifugal coefficient $G_{a}^{K}$ to $-2.0 \mathrm{MHz}$, even if a negligible increasing $(\approx 1.5 \mathrm{kHz})$ of the standard deviation of the fit occurs. At the same time, $G_{a}$ changes from 18734 to $19163 \mathrm{MHz}$. Unfortunately, it is not convenient to simultaneously fit the two $D_{K}$ constants and the $G_{a}^{K}$ coefficient, because correlations are very high, so that $G_{a}^{K}$ assumes a small value with a large uncertainty $\left(G_{a}^{K}=-0.64 \pm 0.14 \mathrm{MHz}\right)$ and the $D_{K}$ constants converge again to values which are fairly different $( \pm 9.4 \%)$ from that of the ground state.

Analysis of the theoretical vibrational wavenumbers obtained from VPT2 computations with the hybrid CC/DFT force field confirms the experimental results. The fundamental bands predicted at 193, 213, and $322 \mathrm{~cm}^{-1}$ agree well with the previously mentioned excited-state vibrational energies derived by intensity ratio measurements, thus allowing the assignment of the lowest energy level to the in-plane $\mathrm{C} \equiv \mathrm{C}-\mathrm{C}$ linear bending of $A^{\prime}$ symmetry $\left(v_{13}\right)$ and of the next one to the $-\mathrm{NH}_{2}$ torsion of $A^{\prime \prime}$ symmetry $\left(v_{21}\right)$. Furthermore, the computed energy difference between the $A^{\prime}$ and $A^{\prime \prime}$ states $\left(20 \mathrm{~cm}^{-1}\right)$ fully justifies a significant $a$-type Coriolis resonance between the two lowest vibrational levels. The third, unperturbed band at $327 \mathrm{~cm}^{-1}$ is assigned to the out-of-plane $\mathrm{C} \equiv \mathrm{C}-\mathrm{C}$ linear bending of $A^{\prime \prime}$ symmetry $\left(v_{20}\right)$.

Inspection of Table 2 shows that the changes in the rotational constants caused by the excitation of the $v_{20}$ vibrational mode (i.e., the contribution of the $\alpha_{20}$ vibration-rotation interaction constants) are well predicted by ab initio calculations. From Table 2, it is apparent that this is also true for the $B$ and $C$ rotational constants of the $v_{13}$ and $v_{21}$ modes, but not for the $A$ constants of both states. This can be explained by considering that the $a$-type Coriolis resonance existing between these two states produces very different values of the ${ }^{A} \alpha_{13}$ and ${ }^{A} \alpha_{21}$ vibration-rotation interaction constants depending on the way it is treated. In the experimental $A$ rotational constants, the resonant term, which is inversely proportional to the small vibrational energy difference $v_{21}-v_{13}$, is removed from the ${ }^{A} \alpha_{13}$ and ${ }^{A} \alpha_{21}$ vibration-rotation interaction constants because the Coriolis coupling is treated variationally. On the contrary, the theoretical calculation makes use of the standard second-order perturbation theory of vibro-rotational interactions (Papoušek \& Aliev 1982), so that a large resonant term of approximately $400 \mathrm{MHz}$ is included (with opposite signs) in the computed values of the $A$ rotational constants of the two interacting states.

The fitted value of the main Coriolis coupling coefficient is $G_{a}=18734 \pm 5 \mathrm{MHz}$, which can be compared with the one derived from computations. The $G_{a}$ coefficient coupling 
Table 3. Comparison between selected spectroscopic constants of the three lowest vibrationally excited states of trans-propargylamine and transaminoacetonitrile.

\begin{tabular}{|c|c|c|c|c|c|c|c|c|}
\hline & & & \multicolumn{3}{|c|}{$\begin{array}{l}\text { Propargylamine } \\
\text { Present work }\end{array}$} & \multicolumn{3}{|c|}{$\begin{array}{c}\text { Aminoacetonitrile } \\
\text { Degli Esposti et al. (2017) }\end{array}$} \\
\hline & & & $v_{13}=1\left(A^{\prime}\right)$ & $v_{21}=1\left(A^{\prime \prime}\right)$ & $v_{20}=1\left(A^{\prime \prime}\right)$ & $v_{11}=1\left(A^{\prime}\right)$ & $v_{18}=1\left(A^{\prime \prime}\right)$ & $v_{17}=1\left(A^{\prime \prime}\right)$ \\
\hline$\alpha^{A}$ & / & $\mathrm{MHz}$ & -15.84 & -119.67 & 51.165 & -33.14 & -119.83 & 102.785 \\
\hline$\alpha^{B}$ & / & $\mathrm{MHz}$ & -13.948 & -7.761 & -2.868 & -15.423 & -8.351 & -3.175 \\
\hline$\alpha^{C}$ & I & $\mathrm{MHz}$ & -5.221 & -3.519 & -5.024 & -5.907 & -3.760 & -5.685 \\
\hline$D_{J}$ & / & $\mathrm{kHz}$ & $2.66689(13)^{a}$ & $2.693125(90)$ & $2.691875(43)$ & $3.048626(79)$ & $3.07047(14)$ & $3.07242(11)$ \\
\hline$D_{J K}$ & / & $\mathrm{kHz}$ & $-49.4855(41)$ & $-51.5737(38)$ & $-51.8353(16)$ & $-52.555(85)$ & $-55.690(85)$ & $-55.0291(22)$ \\
\hline$D_{K}$ & / & $\mathrm{kHz}$ & $650.1(10)$ & $847.29(40)$ & $738.53(27)$ & $649.87(15)$ & $779.29(23)$ & $695.85(19)$ \\
\hline$d_{1}$ & / & $\mathrm{kHz}$ & $-0.612076(27)$ & $-0.615077(74)$ & $-0.612384(32)$ & $-0.674571(61)$ & $-0.675957(75)$ & $-0.672041(62)$ \\
\hline$d_{2}$ & / & $\mathrm{kHz}$ & $-0.032100(18)$ & $-0.028371(17)$ & -0.025814 & $-0.0336185(89)$ & $-0.029711(16)$ & $-0.026762(13)$ \\
\hline$\Delta E^{b}$ & / & $\mathrm{cm}^{-1}$ & \multicolumn{2}{|c|}{$29.3992(2)$} & \multicolumn{4}{|c|}{$34.3173(2)$} \\
\hline$G_{a}$ & / & $\mathrm{MHz}$ & \multicolumn{2}{|c|}{$18734.3(51)$} & \multicolumn{4}{|c|}{$16401.4(46)$} \\
\hline$G_{a}^{J}$ & / & $\mathrm{kHz}$ & \multicolumn{2}{|c|}{$33.0^{c}$} & \multicolumn{4}{|c|}{$33.0(27)$} \\
\hline$G_{a}^{J K}$ & / & $\mathrm{kHz}$ & \multicolumn{2}{|c|}{$-0.03563(88)$} & \multicolumn{4}{|c|}{$-0.03308(54)$} \\
\hline
\end{tabular}

Notes. ${ }^{(a)}$ The quantities in parentheses are standard errors $(1 \sigma)$ in units of the last quoted digit of the value of the constant. ${ }^{(b)}$ Vibrational energy difference between the resonant states of propargylamine $\left(v_{21}-v_{13}\right)$ and aminoacetonitrile $\left(v_{18}-v_{11}\right) . v_{21}$ and $v_{18}$ are the respective upper states. ${ }^{(c)}$ Parameter fixed at the value obtained for aminoacetonitrile (Degli Esposti et al. 2017).

the $v_{13}=1$ and $v_{21}=1$ fundamental levels has the following theoretical expression (Papoušek \& Aliev 1982):

$G_{a}=A_{e} \frac{\omega_{13}+\omega_{21}}{\sqrt{\omega_{13} \omega_{21}}} \zeta_{13,21}^{a}$

where $A_{e}$ is the equilibrium value of the rotational constant $A, \omega_{13}$, and $\omega_{21}$ are the harmonic frequencies of the interacting states, and $\zeta_{13,21}^{a}$ is the corresponding Coriolis coupling constant. Using the best-estimated computed values, namely the $\operatorname{CCSD}(\mathrm{T}) / \mathrm{CBS}+\mathrm{CV}$ equilibrium rotational constant $\left(A_{e}=\right.$ $29767.78 \mathrm{MHz})$, the harmonic wavenumbers as obtained from Eq. (1) $\left(\omega_{13}=192.78 \mathrm{~cm}^{-1}, \omega_{21}=226.09 \mathrm{~cm}^{-1}\right)$, and $\zeta_{13,21}^{a}=$ 0.392 from a composite scheme analogous to Eq. (1), a value of 23412.1 MHz was obtained, which compares qualitatively well to the experimental datum.

The IR spectra of propargylamine (Hamada et al. 1984) and aminoacetonitrile (Bak et al. 1975) are quite similar below $500 \mathrm{~cm}^{-1}$. Three fundamental bands are in that range: two bendings of $A^{\prime}$ and $A^{\prime \prime}$ symmetries, respectively, and the $\mathrm{NH}_{2}$ torsion. Table 3 shows the comparison between selected spectroscopic constants determined for the three lowest vibrationally excited states of trans-propargylamine (present work) and transaminoacetonitrile (Degli Esposti et al. 2017). Vibration-rotation interaction constants $(\alpha)$, quartic centrifugal distortion constants, and parameters related to the Coriolis resonance between the two lowest excited states of each molecule are included in the table. As expected, the vibration-rotation interaction constants and the quartic centrifugal distortion constants exhibit rather parallel trends upon vibrational excitation. The energy separation between the two resonant states is smaller for propargylamine, while the corresponding $G_{a}$ value is greater, so that the perturbation effects produced by the Coriolis resonance are more marked in the rotational spectra of propargylamine than in those of aminoacetonitrile.

The complete list of the analyzed rotational transitions is provided at the CDS. An excerpt is reported in Table 4 for guidance.

\section{Astrophysical implications}

The presence of COMs in the harsh conditions of the ISM has been known for decades (see e.g., Herbst \& van Dishoeck 2009, and references therein). Earlier detections of organic molecules have been restricted to massive star formation regions (e.g., Rubin et al. 1971) but recently they have also been revealed in smaller objects (low-mass protostars, Cazaux et al. 2003), which are considered to be the progenitors of Solar-like planetary systems. Typically, COMs thrive in warm gases $(T>100 \mathrm{~K})$. The large majority of them has been identified toward the Galactic center, in SgrB2 (e.g., Belloche et al. 2013, 2016), but a number of detections in hot-corinos (e.g., van Dishoeck et al. $1995)$ and in the shocked regions generated by protostar ouflows (Codella et al. 2015; Lefloch et al. 2017) have also been reported. At these temperatures, COMs typically have large partition functions thus showing highly crowded rotational spectra. As a result, these individual lines might be weak and therefore hidden in the "line forest" due to the so-called "weeds" or even contributing to it.

In many cases, this line forest consists of rotational lines not only belonging to the vibrational ground state, but also to low-lying vibrational states, which might be significantly populated. Furthermore, lines of different species overlap and blend together thus bringing sensitive spectral observations close to the confusion limit. Ideally, a firm analysis of an unbiased survey would require an accurate modeling of all known molecules, in order to point out blended features and to prevent misassignments.

To help assignment of propargylamine features in unbiased spectral surveys, we provide a list of rest frequencies and transition moments for lines up to $230 \mathrm{GHz}$. Both transitions belonging to the ground state and to the low-lying vibrational states $v_{20}=1, v_{13}=1$, and $v_{21}=1$ are included. To this purpose, the spectroscopic constants of Tables 1 and 2 are used, also taking into account the Coriolis coupling between the $v_{13}=1$ and $v_{21}=1$ states.

For each line, the Einstein spontaneous emission A coefficient can be computed using the formula: 
Table 4. Assignments, measured line positions, and least-squares residuals for the analysed transitions of propargylamine.

\begin{tabular}{|c|c|c|c|c|c|c|c|c|c|c|c|c|c|}
\hline $\begin{array}{c}J^{\prime} \\
(1) \\
\end{array}$ & $\begin{array}{l}K_{a}^{\prime} \\
(2) \\
\end{array}$ & $\begin{array}{l}K_{c}^{\prime} \\
(3) \\
\end{array}$ & $\begin{array}{l}v^{\prime} \\
(4) \\
\end{array}$ & $\begin{array}{l}F^{\prime} \\
(5) \\
\end{array}$ & $\begin{array}{c}J \\
(6) \\
\end{array}$ & $\begin{array}{l}K_{a} \\
(7) \\
\end{array}$ & $\begin{array}{l}K_{c} \\
(8) \\
\end{array}$ & $\begin{array}{c}v \\
(9) \\
\end{array}$ & $\begin{array}{c}F \\
(10) \\
\end{array}$ & $\begin{array}{c}\text { Observed } \\
(11)\end{array}$ & $\begin{array}{c}\text { Obs.-calc. } \\
(12)\end{array}$ & $\begin{array}{c}\text { Uncert. } \\
(13)\end{array}$ & $\begin{array}{c}\text { Weight } \\
(14)\end{array}$ \\
\hline 1 & 0 & 1 & 0 & 1 & 0 & 0 & 0 & 0 & 1 & 8754.9970 & 0.0087 & 0.005 & \\
\hline 1 & 0 & 1 & 0 & 2 & 0 & 0 & 0 & 0 & 1 & 8755.8953 & 0.0026 & 0.005 & \\
\hline 1 & 0 & 1 & 0 & 0 & 0 & 0 & 0 & 0 & 1 & 8757.2501 & 0.0008 & 0.005 & \\
\hline 10 & 1 & 10 & 0 & $*$ & 9 & 1 & 9 & 0 & $*$ & 85170.851 & -0.017 & 0.010 & \\
\hline 10 & 0 & 10 & 0 & $*$ & 9 & 0 & 9 & 0 & $*$ & 86658.130 & 0.001 & 0.010 & \\
\hline 10 & 2 & 9 & 0 & $*$ & 9 & 2 & 8 & 0 & * & 87409.619 & -0.008 & 0.015 & \\
\hline 10 & 7 & 3 & 0 & 10 & 9 & 7 & 2 & 0 & 9 & 87626.025 & -0.021 & 0.020 & 0.50 \\
\hline 10 & 7 & 4 & 0 & 10 & 9 & 7 & 3 & 0 & 9 & 87626.025 & -0.021 & 0.020 & 0.50 \\
\hline 10 & 7 & 3 & 0 & 11 & 9 & 7 & 2 & 0 & 10 & 87626.390 & 0.026 & 0.020 & 0.26 \\
\hline 10 & 7 & 4 & 0 & 11 & 9 & 7 & 3 & 0 & 10 & 87626.390 & 0.026 & 0.020 & 0.26 \\
\hline 10 & 7 & 3 & 0 & 9 & 9 & 7 & 2 & 0 & 8 & 87626.390 & -0.014 & 0.020 & 0.24 \\
\hline 10 & 7 & 4 & 0 & 9 & 9 & 7 & 3 & 0 & 8 & 87626.390 & -0.014 & 0.020 & 0.24 \\
\hline 64 & 4 & 61 & 0 & $*$ & 63 & 4 & 60 & 0 & $*$ & 553940.631 & -0.004 & 0.020 & \\
\hline 65 & 2 & 64 & 0 & $*$ & 64 & 1 & 63 & 0 & $*$ & 549064.979 & -0.024 & 0.020 & \\
\hline 9 & 1 & 9 & 1 & $*$ & 8 & 1 & 8 & 1 & $*$ & 76816.540 & 0.007 & 0.015 & \\
\hline 9 & 0 & 9 & 1 & $*$ & 8 & 0 & 8 & 1 & $*$ & 78276.573 & 0.003 & 0.015 & \\
\hline 9 & 2 & 8 & 1 & $*$ & 8 & 2 & 7 & 1 & $*$ & 78863.436 & 0.007 & 0.020 & \\
\hline 10 & 7 & 3 & 1 & 10 & 9 & 7 & 2 & 1 & 9 & 87814.069 & -0.011 & 0.020 & 0.50 \\
\hline 10 & 7 & 4 & 1 & 10 & 9 & 7 & 3 & 1 & 9 & 87814.069 & -0.011 & 0.020 & 0.50 \\
\hline 10 & 7 & 3 & 1 & 11 & 9 & 7 & 2 & 1 & 10 & 87814.429 & 0.031 & 0.020 & 0.26 \\
\hline 10 & 7 & 4 & 1 & 11 & 9 & 7 & 3 & 1 & 10 & 87814.429 & 0.031 & 0.020 & 0.26 \\
\hline 10 & 7 & 3 & 1 & 9 & 9 & 7 & 2 & 1 & 8 & 87814.429 & -0.008 & 0.020 & 0.24 \\
\hline 10 & 7 & 4 & 1 & 9 & 9 & 7 & 3 & 1 & 8 & 87814.429 & -0.008 & 0.020 & 0.24 \\
\hline 34 & 6 & 29 & 1 & $*$ & 33 & 6 & 28 & 1 & $*$ & 299155.896 & 0.002 & 0.020 & \\
\hline 34 & 6 & 28 & 1 & $*$ & 33 & 6 & 27 & 1 & $*$ & 299171.746 & -0.003 & 0.020 & \\
\hline 39 & 1 & 39 & 1 & $*$ & 38 & 1 & 38 & 1 & $*$ & 327901.796 & -0.001 & 0.020 & \\
\hline 39 & 0 & 39 & 1 & $*$ & 38 & 0 & 38 & 1 & $*$ & 327918.765 & -0.016 & 0.020 & \\
\hline 9 & 1 & 9 & 2 & $*$ & 8 & 1 & 8 & 2 & $*$ & 76774.271 & 0.006 & 0.015 & \\
\hline 9 & 0 & 9 & 2 & $*$ & 8 & 0 & 8 & 2 & $*$ & 78241.811 & 0.002 & 0.015 & \\
\hline 9 & 2 & 8 & 2 & $*$ & 8 & 2 & 7 & 2 & $*$ & 78799.091 & -0.007 & 0.020 & \\
\hline 10 & 7 & 3 & 2 & 10 & 9 & 7 & 2 & 2 & 9 & 87741.521 & -0.018 & 0.020 & 0.50 \\
\hline 10 & 7 & 4 & 2 & 10 & 9 & 7 & 3 & 2 & 9 & 87741.521 & -0.018 & 0.020 & 0.50 \\
\hline 10 & 7 & 3 & 2 & 11 & 9 & 7 & 2 & 2 & 10 & 87741.859 & 0.022 & 0.020 & 0.26 \\
\hline 10 & 7 & 4 & 2 & 11 & 9 & 7 & 3 & 2 & 10 & 87741.859 & 0.022 & 0.020 & 0.26 \\
\hline 10 & 7 & 3 & 2 & 9 & 9 & 7 & 2 & 2 & 8 & 87741.859 & -0.015 & 0.020 & 0.24 \\
\hline 10 & 7 & 4 & 2 & 9 & 9 & 7 & 3 & 2 & 8 & 87741.859 & -0.015 & 0.020 & 0.24 \\
\hline 34 & 6 & 29 & 2 & $*$ & 33 & 6 & 28 & 2 & $*$ & 298862.541 & 0.004 & 0.020 & \\
\hline 34 & 6 & 28 & 2 & $*$ & 33 & 6 & 27 & 2 & $*$ & 298877.335 & -0.002 & 0.020 & \\
\hline 42 & 0 & 42 & 2 & $*$ & 41 & 1 & 41 & 2 & $*$ & 352684.374 & 0.013 & 0.020 & \\
\hline 10 & 1 & 10 & 3 & $*$ & 9 & 1 & 9 & 3 & $*$ & 85262.099 & 0.000 & 0.010 & \\
\hline 10 & 2 & 9 & 3 & $*$ & 9 & 2 & 8 & 3 & $*$ & 87489.609 & -0.002 & 0.015 & \\
\hline 10 & 7 & 3 & 3 & 10 & 9 & 7 & 2 & 3 & 9 & 87704.679 & -0.020 & 0.020 & 0.50 \\
\hline 10 & 7 & 4 & 3 & 10 & 9 & 7 & 3 & 3 & 9 & 87704.679 & -0.020 & 0.020 & 0.50 \\
\hline 10 & 7 & 3 & 3 & 11 & 9 & 7 & 2 & 3 & 10 & 87705.039 & 0.022 & 0.020 & 0.26 \\
\hline 10 & 7 & 4 & 3 & 11 & 9 & 7 & 3 & 3 & 10 & 87705.039 & 0.022 & 0.020 & 0.26 \\
\hline 10 & 7 & 3 & 3 & 9 & 9 & 7 & 2 & 3 & 8 & 87705.039 & -0.018 & 0.020 & 0.24 \\
\hline 10 & 7 & 4 & 3 & 9 & 9 & 7 & 3 & 3 & 8 & 87705.039 & -0.018 & 0.020 & 0.24 \\
\hline 34 & 6 & 29 & 3 & $*$ & 33 & 6 & 28 & 3 & $*$ & 298722.618 & -0.010 & 0.020 & \\
\hline 34 & 6 & 28 & 3 & $*$ & 33 & 6 & 27 & 3 & $*$ & 298737.942 & 0.029 & 0.020 & \\
\hline 30 & 0 & 30 & 3 & $*$ & 29 & 1 & 29 & 3 & $*$ & 252480.569 & -0.022 & 0.020 & \\
\hline
\end{tabular}

Notes. Column content: (1-3) upper state rotational quantum numbers $J^{\prime}, K_{a}^{\prime}, K_{c}^{\prime}$; (4) upper state vibrational label; (5) upper state total quantum number $F^{\prime} ;(6-8)$ lower state rotational quantum numbers $J, K_{a}, K_{c}$; (9) lower state vibrational label; (10) lower state total quantum number $F$; (11) measured line frequency (MHz); (12) least-squares residual (MHz); (13) assumed uncertainty (MHz); (14) relative weights for blended transitions. Asterisks in Cols. (5) and (10) indicate completely unresolved hyperfine structure. Vibrational labels are 0 for ground state, 1 for $v_{13}=1,2$ for $v_{21}=1$, and 3 for $v_{20}=1$. The full table is available at the CDS. 

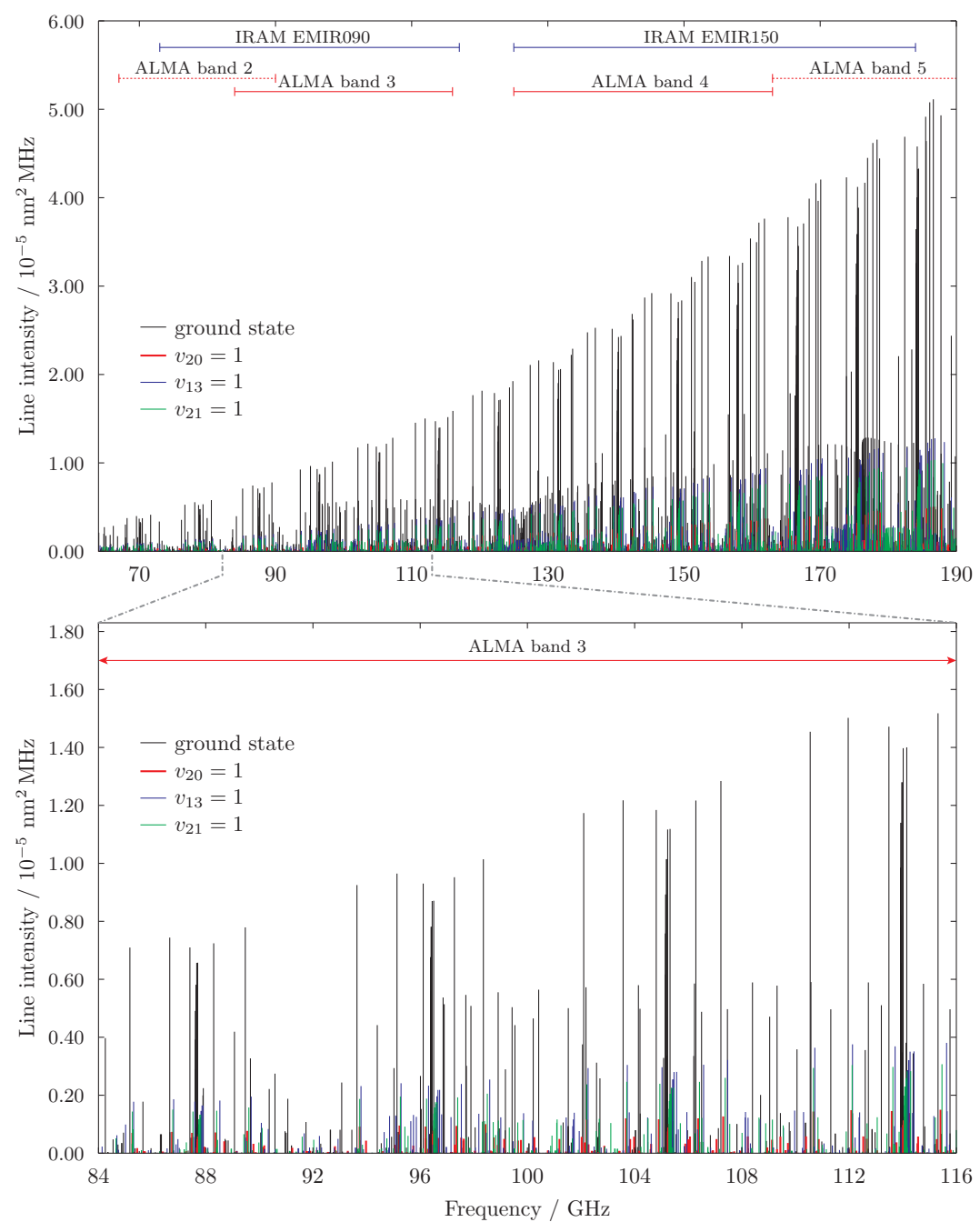

Fig. 3. Upper panel: stick spectra of ground, $v_{20}=1$, $v_{13}=1$, and $v_{21}=1$ states in the $66-190 \mathrm{GHz}$ frequency range. Lower panel: expansion of the $84-116 \mathrm{GHz}$ region. Intensities are computed at $200 \mathrm{~K}$.

$A_{i j}=\frac{16 \pi^{3} v^{3}}{3 \epsilon_{0} h c^{3}} \frac{1}{(2 J+1)} S_{i j} \mu^{2}$,

where all quantities are expressed in SI units and the line strengths $S_{i j} \mu^{2}$ are obtained projecting the squared rotation matrix onto the basis set that diagonalizes the rotational Hamiltonian (Gordy \& Cook 1984). The spectral calculations were performed in the $25 \leq v \leq 250 \mathrm{GHz}$ frequency interval selecting lines with $E_{u} / k<500 \mathrm{~K}$ and Einstein's $A>1 \times 10^{-8} \mathrm{~s}$. A further filter based on the prediction uncertainty was applied in order to select all the lines for which $1 \sigma<0.050 \mathrm{MHz}$, corresponding to $0.03 \mathrm{~km} \mathrm{~s}^{-1}$ at $500 \mathrm{GHz}$. The procedure yielded a sample of $3833,769,732$, and 83 lines for the ground, $v_{21}=$ $1, v_{13}=1$, and $v_{20}=1$ states, respectively. Generally speaking, the predicted frequency uncertainties are model-dependent, that is, additional Hamiltonian terms, which are indeterminable from a given set of experimental data, can cause their increase. However, the spectroscopic constants used in the calculation described above have been determined from measurements extending up to $555 \mathrm{GHz}$, we thus expect that the precision of the resulting rest frequencies is reliably estimated.

At the typical temperature of a hot molecular core (e.g., SgrB2, $T \sim 200 \mathrm{~K}$, Belloche et al. 2013), propargylamine produces a crowded emission spectrum including many features belonging to some low-lying vibrational excited states. This is illustrated in Fig. 3, where the stick spectra of the ground, $v_{20}=1, v_{13}=1$, and $v_{21}=1$ states are plotted in the $66-190 \mathrm{GHz}$ frequency range. This interval matches the frequency span of the EMIR E090 and EMIR150 receivers installed at the IRAM $30 \mathrm{~m}$ telescope, and the ALMA bands from 2 to 5. The lower panel expands the $84-116 \mathrm{GHz}$ region covered by ALMA band 3, that is the band used in the EMoCA survey of SgrB2(N) performed during ALMA Cycle 0 and Cycle 1 (Belloche et al. 2016). The fact that several lines corresponding to the excited vibrational states show non-negligible intensity indicates that propargylamine might increase the confusion limit.

\section{Conclusions}

This paper presents the results of a comprehensive investigation of the rotational spectrum of propargylamine extending well into the submm-wave region. The rotational spectrum of the vibrational ground state has been studied over a large frequency range, leading to greatly improved spectroscopic constants with respect to those available from earlier $\mathrm{cm}$-wave measurements (Cervellati et al. 1977). The complete set of sextic centrifugaldistortion constants has been accurately determined for the first time, together with a few octic terms. Additionally, pulsed-jet FTMW measurements made it possible to perform an accurate analysis of the ${ }^{14} \mathrm{~N}$-quadrupole hyperfine structure.

The rotational spectra of the three lowest vibrational excited states have also been identified for the first time. Their energies have been approximately evaluated by means of intensity ratio measurements, which provided values not completely consistent with the vibrational bands assigned in Hamada et al. (1984). Theoretical calculations of vibrational energies agree much 
better with the newly estimated values (i.e., a fundamental level exists just below $200 \mathrm{~cm}^{-1}$ ) but there are not two nearly degenerate fundamental vibrations just above $300 \mathrm{~cm}^{-1}$.

Unlike the findings of Andrews et al. (1994) from the $10 \mu \mathrm{m}$ infrared spectrum, no tunneling doublets, indicative of amine proton interchange, were observed either in the FTMW groundstate spectrum or in the excited states spectra studied in this work. However, we note that in rotational studies, information about the tunnelling effect must be inferred from minor differences between the effective rotational constants of the doublet states. These differences are typically small even when the tunnelling energy splitting is sizable.

Propargylamine is structurally similar to well-known interstellar species containing the acetylenic group (e.g., $\mathrm{CH}_{3} \mathrm{C} \equiv \mathrm{CH}$, $\mathrm{HC} \equiv \mathrm{C}-\mathrm{CN}$, and $\left.\mathrm{HC} \equiv \mathrm{C}-\mathrm{CNH}^{+}\right)$or the amino group $\left(\mathrm{CH}_{3} \mathrm{NH}_{2}\right.$ and $\mathrm{NC}-\mathrm{CH}_{2}-\mathrm{NH}_{2}$ ). Propargylamine itself can therefore be considered a molecule of potential astrochemical interest. The sets of spectroscopic constants obtained in this study allow for very accurate predictions of the rotational frequencies of propargylamine in its vibrational ground state and even of the weaker rotational features due to the low-lying vibrational states, thus offering a valuable support for a targeted search for this molecule in the ISM.

Acknowledgements. This work was supported by Italian MIUR (PRIN 2012 "STAR: Spectroscopic and computational Techniques for Astrophysical and atmospheric Research"; PRIN 2015 "STARS in the CAOS (Simulation Tools for Astrochemical Reactivity and Spectroscopy in the Cyberinfrastructure for Astrochemical Organic Species)"), by the University of Bologna (RFO funds), the Deutsche Forschungsgemeinschaft (DFG), the Land Niedersachsen, and in Shanghai by the National Natural Science Foundation of China (Grant No. 91641128). The SMART@SNS Laboratory (http://smart.sns.it) is gratefully acknowledged for the use of computer resources. The support of the COST CMTS-Actions CM1405 (MOLIM: MOLecules In Motion) and CM1401 (Our Astro-Chemical History) is acknowledged.

\section{References}

Ali, A., Sittler Jr., E. C., Chornay, D., Rowe, B. R., \& Puzzarini, C. 2015, Planet Space Sci., 109-110, 46

Andrews, A. M., Fraser, G. T., \& Pate, B. H. 1994, J. Phys. Chem., 98, 9979

Andrews, A. M., Fraser, G. T., \& Pate, B. H. 1998, J. Chem. Phys., 109, 4290

Bak, B., Hansen, E. L., Nicolaisen, F. M., \& Nielsen, O. F. 1975, Can. J. Phys., 53,2183

Balucani, N. 2012, Chem. Soc. Rev., 41, 5473

Barone, V., Biczysko, M., Bloino, J., \& Puzzarini, C. 2013, Phys. Chem. Chem. Phys., 15, 10094

Barone, V., Biczysko, M., \& Bloino, J. 2014a, Phys. Chem. Chem. Phys., 16, 1759

Barone, V., Biczysko, M., Bloino, J., \& Puzzarini, C. 2014b, J. Chem. Phys., 141, 034107

Barone, V., Biczysko, M., Bloino, J., et al. 2015a, J. Chem. Theory Comput., 11, 4342

Barone, V., Biczysko, M., \& Puzzarini, C. 2015b, Acc. Chem. Res., 48, 1413

Belloche, A., Menten, K. M., Comito, C., et al. 2008, A\&A, 482, 179

Belloche, A., Müller, H. S., Menten, K. M., Schilke, P., \& Comito, C. 2013 , A\&A, 559, A47

Belloche, A., Müller, H. S. P., Garrod, R. T., \& Menten, K. M. 2016, A\&A, 587, A91

Biczysko, M., Panek, P., Scalmani, G., Bloino, J., \& Barone, V. 2010, J. Chem. Theory Comput., 6, 2115

Biczysko, M., Bloino, J., \& Puzzarini, C. 2018, WIREs Comput. Mol. Sci., 8 , e1349

Bisschop, S. E., Fuchs, G. W., Van Dishoeck, E. F., \& Linnartz, H. 2007, A\&A, 474, 1061

Bizzocchi, L., Dore, L., Degli Esposti, C., \& Tamassia, F. 2016, ApJ, 820, L26
Bizzocchi, L., Tamassia, F., Laas, J., et al. 2017, ApJS, 233, 11

Bloino, J., Biczysko, M., \& Barone, V. 2015, J. Phys. Chem. A, 119, 11862

Bloino, J., Baiardi, A., \& Biczysko, M. 2016, Int. J. Quantum Chem., 116, 1543

Bolton, K., Owen, N. L., \& Sheridan, J. 1968, Nature, 217, 164

Bossa, J.-B., Borget, F., Duvernay, F., Theulé, P., \& Chiavassa, T. 2008, J. Phys. Chem. A, 112, 5113

Cazaux, S., Tielens, A. G. G. M., Ceccarelli, C., et al. 2003, ApJ, 593, L51

Ceccarelli, C., Loinard, L., Castets, A., et al. 2001, A\&A, 372, 998

Cervellati, R., Caminati, W., Degli Esposti, C., \& Mirri, A. M. 1977, J. Mol. Spectr., 66, 389

Codella, C., Fontani, F., Ceccarelli, C., et al. 2015, MNRAS, 449, L11

Degli Esposti, C., Dore, L., \& Bizzocchi, L. 2014, A\&A, 565, A66

Degli Esposti, C., Dore, L., Melosso, M., et al. 2017, ApJS, 230, 26

Dunning Jr., T. H. 1989, J. Chem. Phys., 90, 1007

Frisch, M. J., Trucks, G. W., Schlegel, H. B., et al. 2016, Gaussian 16 Revision A.03, Gaussian Inc., Wallingford CT

Fuchs, G. W., Cuppen, H. M., Ioppolo, S., et al. 2009, A\&A, 505, 629

Garrod, R. T., Wakelam, V., \& Herbst, E. 2007, A\&A, 467, 1103

Gauss, J., \& Stanton, J. F. 1997, Chem. Phys. Lett., 276, 70

Gordy, W., \& Cook, R. L. 1984, Microwave Molecular Spectra, 3rd edn. (New York: Wiley)

Grabow, J.-U., \& Stahl, W. 1990, Z. Naturforsch., 45a, 1043

Grabow, J.-U., Stahl, W., \& Dreizler, H. 1996, Rev. Sci. Instrum., 67, 4072

Grimme, S. 2006, J. Chem. Phys., 124, 034108

Grimme, S., Antony, J., Ehrlich, S., \& Krieg, H. 2010, J. Chem. Phys., 132, 154104

Grimme, S., Ehrlich, S., \& Goerigk, L. 2011, J. Comput. Chem., 32, 1456

Hamada, Y., Tsuboi, M., Nakata, M., \& Tasumi, M. 1984, J. Mol. Spectr., 107, 269

Heckert, M., Kállay, M., \& Gauss, J. 2005, Mol. Phys., 103, 2109

Heckert, M., Kállay, M., Tew, D. P., Klopper, W., \& Gauss, J. 2006, J. Chem. Phys., 125, 044108

Herbst, E., \& van Dishoeck, E. F. 2009, Annu. Rev. Astron. Astrophys., 47, 427

Holtom, P. D., Bennett, C. J., Osamura, Y., Mason, N. J., \& Kaiser, R. I. 2005, ApJS, 626, 940

Ioppolo, S., Cuppen, H. M., Van Dishoeck, E. F., \& Linnartz, H. 2010, MNRAS, 410, 1089

Kim, Y. S., \& Kaiser, R. I. 2011, ApJ, 729, 68

Krohn, B., Ermler, W., \& Kern, C. 1974, J. Chem. Phys., 60, 22

Lee, C.-W., Kim, J.-K., Moon, E.-S., Minh, Y. C., \& Kang, H. 2009, ApJ, 697, 428

Lefloch, B., Ceccarelli, C., Codella, C., et al. 2017, MNRAS, 469, L73

Møller, C., \& Plesset, M. S. 1934, Phys. Rev., 46, 618

Motoki, Y., Tsunoda, Y., Ozeki, H., \& Kobayashi, K. 2013, ApJS, 209, 23

Nielsen, H. H. 1951, Rev. Mod. Phys., 23, 90

Papoušek, D., \& Aliev, M. R. 1982, Molecular Vibrational-Rotational Spectra (Elsevier Science Ltd)

Pickett, H. M. 1991, J. Mol. Spectr., 148, 371

Puzzarini, C. 2013, Phys. Chem. Chem. Phys., 15, 6595

Puzzarini, C., Heckert, J., \& Gauss, J. 2008, J. Chem. Phys., 128, 194108

Puzzarini, C., Biczysko, M., Barone, V., et al. 2014, J. Phys. Chem. Lett., 5, 534

Raghavachari, K., Trucks, G. W., Pople, J. A., \& Head-Gordon, M. 1989, Chem. Phys. Lett., 157, 479

Rubin, R. H., Swenson, Jr. G. W., Benson, R. C., Tigelaar, H. L., \& Flygare, W. H. 1971, ApJ, 169, L39

Snyder, L. E., \& Bull, D. 1973, Nature Phys. Sci., 243, 45

Stanton, J. F., Gauss, J., Harding, M. E., \& Szalay, P. G. 2011, CFour A quantum chemical program package. For the current version, see http: //www. cfour.de

Theulé, P., Borget, F., Mispelaer, F., et al. 2011, A\&A, 534, A64

Tielens, A. G. G. M., \& Hagen, W. 1982, A\&A, 114, 245

Tielens, A. G. G. M., \& Charnley, S. B. 1997, in Planetary and Interstellar Processes Relevant to the Origins of Life (Springer), 23

van Dishoeck, E. F., Blake, G. A., Jansen, D. J., \& Groesbeck, T. D. 1995, ApJ, 447,760

Watanabe, N., \& Kouchi, A. 2002, ApJ, 567, 651

Watson, J. K. G. 1977, in Vibrational Spectra and Structure, ed. J. R. Durig (New York: Marcel Dekker), 6, 1

Woon, D. E., \& Dunning Jr., T. H. 1995, J. Chem. Phys., 103, 4572 